\title{
Proteomic Analysis of Banana Vascular Sap Provides Insight Into Resistance Mechanisms to Fusarium Oxysporum F. Sp. Cubense Tropical Race 4
}

\section{Lei Zhang}

Yunnan Academy of Agricultural Sciences

Lina Liu

Yunnan Academy of Agricultural Sciences

Shu Li

Yunnan Academy of Agricultural Sciences

Alberto Cenci

Bioversity International

Mathieu Rouard

Bioversity International

\section{Sebastien Carpentier}

Bioversity International

\section{Tingting Bai}

Yunnan Academy of Agricultural Sciences

\section{Shengtao Xu}

Yunnan Academy of Agricultural Sciences

\section{Huacai Fan}

Yunnan Academy of Agricultural Sciences

Kesuo Yin

Yunnan Academy of Agricultural Sciences

\section{Ping He}

Yunnan Academy of Agricultural Sciences

\section{Yunyue Wang}

Yunnan Agriculture University: Yunnan Agricultural University

\section{Weihua Tang}

Shanghai Institute of Plant Physiology and Ecology: Institute of Plant Physiology and Ecology Shanghai Institutes for Biological Sciences

\section{Sijun Zheng ( $\nabla$ s.zheng@cgiar.org )}

Key Laboratory of Green Prevention and Control of Agricultural Transboundary Pests of Yunnan Province, Agricultural Environment and Resources Institute, Yunnan Academy of Agricultural Sciences, 
Kunming 650205, Yunnan, China https://orcid.org/0000-0003-1550-3738

\section{Research article}

Keywords: Banana, Vascular sap, Proteome, Fusarium wilt, Tropical race 4

Posted Date: March 13th, 2021

DOI: https://doi.org/10.21203/rs.3.rs-307891/v1

License: (c) (i) This work is licensed under a Creative Commons Attribution 4.0 International License. Read Full License 


\section{Abstract}

Background: Fusarium oxysporum f. sp. cubense tropical race 4 (Foc TR4) is the causal agent of Fusarium wilt, and is the most destructive soil-borne and vascular invasive fungus of banana. The sap circulating in vascular cells transports proteins including those that might be involved in diseaseresistance processes. However, there is no research to analyze changes in banana vascular sap protein response to TR4 to date.

Results: To gain an integrated understanding of differential protein abundance in banana vascular sap during TR4 infection, we performed a comparative proteomic analysis of vascular sap of the resistant 'Pahang' and the susceptible 'Brazilian' bananas inoculated with TR4. We identified 129 differential expression proteins (DEPs) between resistant and susceptible tested combinations. Of these DEPs, hypersensitive-induced response protein 1 (HIR1) and E3 ubiquitin ligase (E3) decreased in abundance in Pahang with no change in Brazilian under TR4 infection; chalcone isomerase (CHI) and glycine-rich RNAbinding protein (GRP) increased in abundance in Pahang but no significant changes in Brazilian under TR4 infection; carboxylesterase (CXE) and GDSL lipase (GLIP) were specifically in higher abundance in Pahang response to TR4 compared to that of Brazilian. It suggested that these proteins played important roles in bananas against TR4.

Conclusions: Our study identified 129 DEPs in vascular sap between resistant and susceptible tested combinations. Of which, HIR1, E3, CHI, GRP, CXE and GLIP played important roles in bananas response to TR4. To our knowledge, this is first report to analyze changes in banana vascular sap proteins in response to TR4, which help us to explore the molecular mechanisms of banana defense to Fusarium wilt.

\section{Background}

Banana (Musa spp.) is an important fruit and major staple food, and is widely grown in tropical and subtropical countries with an annual output of 145 million tons worldwide [1]. The Cavendish subgroup (AAA genome group), derived from a single triploid genotype, represents approximately $45 \%$ of all productions [2]. However, global banana industry is threatened by Fusarium wilt caused by Fusarium oxysporum f. sp. cubense tropical race 4 (Foc TR4) [3, 4]. TR4 affects many banana cultivars [5] and the rapid expansion of TR4 worldwide is worrisome [6-8]. Fusarium wilt of banana is indeed disastrous to producers. The susceptible cultivars cannot be replanted once the pathogen invaded the field, because its chlamydospores survive for decades in the soil even absence of banana [9]. Recently, Latin America was put on alert after TR4 presence was reported in Colombia [10, 11].

Unfortunately, few effective measures can be used to manage this disease, except the usage of a resistant cultivar [12]. Breeding resistant cultivars is a solution for effective disease management [13]. Banana conventional breeding is difficult because most cultivars are triploid, sterile and parthenocarpic. 
Genetically modification of a susceptible commercial banana is a promising alternative for banana improvement [14-16]. However, defense mechanisms of banana against TR4 are not well understood.

TR4 is the most destructive soil-borne and vascular invasive fungus. It invades root vascular bundles and extends upward to the aerial parts. We previously investigated transcriptomics in the corm to identify the pathways involved in the resistance [17]. Proteomics, complementary to transcriptomics, can provide insights into complex biological processes in banana [18-20]. Large-scale studies of proteomics previously focused on dissecting interactions between bananas and Foc [21-23]. The proteins related to PR response, cell wall strengthening and antifungal compound synthesis were involved in banana defense to TR4 [22]. $\beta$-1,3-glucanase and chitinase were reported to function in banana against TR4 at the early defense stage [21]. The expression patterns of proteins related with cell cytoskeleton, natural killer cell mediated cytotoxicity and lipid signaling were different in banana during Foc1 and Foc4 infection, suggesting these proteins participated in mediating different resistance to Foc1 and Foc4 in banana cultivar 'Brazilian' [23]. These studies help us to understand the defense mechnism of banana against TR4.

Plants transport signal molecules as well as water and minerals over long distance via the vascular bundles [24]. The signal molecules are vital for plant adaption to abiotic and biotic stress $[25,26]$. The vascular sap proteomics have been applied to characterize the processes associated with plant defense to Fusarium wilt [27-31]. However, to our knowledge, there is no research to analyze changes in banana vascular sap proteins response to TR4 to date. In this study, we performed a comparative proteomics analysis of vascular sap in resistant and susceptible bananas inoculated with TR4. 129 DEPs were identified between resistant and susceptible tested combinations, among which HIRP1, E3, CHI, GRP, CXE and GLIP involved in banana defense against TR4. This study provides integrated insight into the resistant mechanism of banana against Fusarium wilt.

\section{Results}

Vascular saps of Pahang (resistant) and Brazilian (susceptible) inoculated with TR4 or mock at $14 \mathrm{dpi}$ were collected for comparative proteomic analysis. A total of 261,038 spectra were acquired through iTRAQ quantitative proteomics analysis, among which 31,450 spectra were matched to 6,503 peptides and 1036 proteins.

\section{The vascular proteome of banana}

All identified proteins $(1,036)$ were conducted functional analysis, of which 938,923 and 779 proteins were annotated with $\mathrm{COG}, \mathrm{GO}$ and KEGG databases, respectively.

In terms of COG (Fig. 2), 938 proteins were assigned into 23 functional categories. Of which 'posttranslational modification, protein turnover, chaperones', 'energy production and conversion' and 'carbohydrate transport and metabolism' were the top 3 largest categories. Related to plant defense were 'lipid transport and metabolism', 'cell wall/membrane/envelope biogenesis', 'secondary metabolites 
biosynthesis, transport and catabolism', 'signal transduction mechanisms' and 'defense mechanism' categories.

In terms of GO (Fig. 3), 923 proteins were assigned into $43 \mathrm{GO}$ terms, and divided into 3 groups, including biological process, cellular component and molecular function. 'Response to stimulus', 'signaling', 'detoxification', 'immune system process' and 'antioxidant activity' were usually regarded to relate to disease resistance among molecular functional groups.

In terms of KEGG (Fig. 4), 779 proteins were assigned into 18 pathways, and divided into 5 categories. Among which the most abundant protein pathways were 'carbohydrate metabolism', 'signal transduction', 'transport and catabolism', 'folding, sorting and degradation' and 'environmental adaptation'. The proteins involved in 'signal transduction', 'environmental adaptation', 'biosynthesis of secondary metabolites' and 'lipid metabolism' might play roles in plant defense against pathogen infection (Supplementary Table 1), such as nucleoside diphosphate kinases, pathogenesis-related protein 1, coronatine-insensitive protein homolog, caffeoyl-CoA 0-methyltransferase, calcium-binding protein CML7, calcium-dependent protein kinase, cinnamoyl-CoA reductases, cinnamyl alcohol dehydrogenase, phenylalanine ammonia-lyase , 4coumarate-CoA ligase, peroxidases, chalcone synthase, flavonoid 3',5'-hydroxylases, dihydroflavonol-4reductase and long chain acyl-CoA synthetases, phospholipase D a1.

\section{Differential expression proteins analysis}

A total of 129 unique DEPs were identified in the 4 pairwise comparisons between mock and inoculated in resistant and susceptible genotypes (Fig. $5 \mathrm{a}$ and $5 \mathrm{~b}$ ), for which we performed expression and function annotation analysis (Supplementary Table 2). A number of 29 DEPs were obtained inoculated samples for mock samples (P_dpi vs P_mock, B_dpi vs B_mock). 19 DEPs (Fig. 5c) were changed significantly in Pahang against TR4 attack (P_dpi vs P_mock). 11 DEPs (Fig. 5d) were changed significantly in Brazilian responding to TR4 (B_dpi vs B_mock). Only one protein (fumarylacetoacetase, XP_009398768.1) increased in both infected bananas. Hypersensitive-induced response protein 1 and E3 ubiquitin ligases decreased in abundance in Pahang under TR4 infection. Chalcone-flavonone isomerase and glycine-rich RNA-binding proteins increased in abundance in Pahang response against TR4 infection.

To further explore the associated resistance mechanisms of Pahang, the DEPs were identified by comparing TR4 inoculated Pahang with that of Brazilian (P_dpi vs B_dpi). A number of 52 DEPs were obtained, of these were 31 up-regulated DEPs and 21 down-regulated DEPs but of 26 DEPs (Fig. $5 e$ ) are unique when excluding DEPs in common with (P_mock vs B_mock). It contains the Carboxylesterases and GDSL esterase/lipases which increased in abundance in TR4 inoculated Pahang compared with that of Brazilian.

\section{Discussion}

TR4 is a vascular-invading fungus, and it colonizes in the vascular system of banana and completes its life cycle [32]. Vascular sap contains macromolecules, such as proteins, involved in disease-resistance 
processes $[27,28,31]$. To gain an integrated understanding of the changes of banana vascular sap proteins during TR4 infection, we performed a comparative proteomic analysis of vascular sap in resistant diploid 'Pahang' and susceptible triploid 'Brazilian' inoculated with TR4 at $14 \mathrm{dpi}$. The amount of fungal biomass and the degree of necrosis in Pahang tissues were significantly less than their levels in Brazilian at $14 \mathrm{dpi}[17,33]$.

\section{Signal transduction}

Signal transduction pathways are responsible for induction of plant defense against pathogen [34, 35], such as mitogen-activated protein kinase (MAPK) cascades and plant hormone signals [36]. Once plant perceives the invading pathogen, the activation of MAPKs is one of the earliest signaling events [37]. In the present study, we found 10 proteins related with MAPK signaling pathway-plant (Supplementary Table 1), such as nucleoside diphosphate kinases known to be an inducer of MPK3/6 expression through phosphorylation leading to hypersensitive response (HR) cell death in plant response to pathogen attack [38].

Salicylic acid (SA) and jasmonic acid (JA) are essential hormone signals of plant immunity [39]. SA and JA antagonize each other [40]. It is generally considered that SA enhances resistance to biotrophs, while JA is effectively against necrotrophs and insects [41, 42]. Fusarium oxysporum was classified as hemibiotrophs [43]. SA metabolism activation and signal transduction, and JA induced defense responses improved banana resistance to TR4 $[44,45]$. In this study, we identified 2 proteins associated with SA and JA signaling (Supplementary table 1), including pathogenesis-related protein 1 (PR1), a marker for systemic acquired resistance (SAR) from SA signaling pathway [46]; and coronatineinsensitive protein homolog (COI1), a key regulator for JA-dependent induced systemic resistance (ISR) $[41,47]$. Further research is needed to determine whether SA-dependent SAR and JA-dependent ISR are simultaneously activated in banana.

\section{Environmental adaptation}

In their natural habitats, plants are threatened by various abiotic and biotic stress. Over the evolutionary course during plant-pathogen interaction, plants have developed multi-layered innate immune system to defend against pathogen. The preliminary layer of immune is pathogen-associated molecular pattern (PAMP) perceived by pathogen recognition receptors (PRRs), and induces a series of physiological changes leading to PAMP-triggered immunity (PTI) [48]. These physiological changes include reactive oxygen species (ROS) bursts and calcium $\left(\mathrm{Ca}^{2+}\right)$ concentrations changes $[49,50] . \mathrm{Ca}^{2+}$ acts as an important second messenger whose concentration is sensed by $\mathrm{Ca}^{2+}$-binding proteins, such as calciumdependent protein kinase (CDPK) and calcium-binding protein CML7 (CaMCML) that we detected in the banana vascular sap (Supplementary Table 1). It initiates downstream signaling processes [51], such as hypersensitive response and cell wall reinforcement.

\section{Biosynthesis of secondary metabolites}


Plant secondary metabolites contribute to all aspects in plant and pathogen interactions [52]. In the biosynthesis of secondary metabolites, phenylpropanoid and flavonoid biosynthesis have been proved to encompass a wide range of constitute and inducible immunity through lignin and phytoalexin synthesis [53]. In our experiment, we found 21 proteins involved in the phenylpropanoid biosynthesis (Supplementary Table 1). We detected synthetic enzymes of lignin leading to strengthen cell walls [17], including phenylalanine ammonia-lyase (PAL), 4-coumarate-CoA ligase (C4L), 2 cinnamoyl-CoA reductases (CCR), cinnamyl alcohol dehydrogenase (CAD) and 3 peroxidases (POD) (Supplementary Table 1). In addition, caffeoyl-CoA 0-methyltransferase (CCOAOMT) associated with lignin production resulting in quantitative resistance to multiple pathogens [54], was found. 13 proteins were assigned to flavonoid biosynthesis, such as chalcone synthase (CHS) as the gatekeeper of flavonoid biosynthesis which can help plant to produce more flavonoids, isoflavonoid-type phytoalexins [55], three P450 enzyme flavonoid 3',5'-hydroxylase and dihydroflavonol-4-reductase (DFR) as precursors for the production of catechins and pro-anthocyanidins involved in plant resistance [56].

\section{Lipid metabolism}

Lipids and fatty acids involved in lipid metabolism are considered as signal transduction mediators of plant disease resistance $[57,58]$. We found two long chain acyl-CoA synthetases (LACS) involved in fatty acids metabolism known to act in the synthesis of cutin to confer plant resistance to fungal pathogen $[59,60]$. Additionally phospholipases D a1 (PLDa1) involved in lipid metabolism which promote phosphatidic acid and ROS [61] were detected in vascular sap (Supplementary Table 1).

\section{Differential protein expression response to TR4 infection}

Among the 129 DEPs identified in 4 possible pairs between resistant and susceptible combinations, only 19 and 11 DEPs were identified in P_dpi vs P_mock and B_dpi vs B_mock, respectively (Supplementary Table 2). It suggests that TR4 did not induce highly dramatic changes in the overall vascular sap proteome. This result was similar to the proteomic analysis of phloem sap in melon defense against viral infection [62]. Nevertheless, these DEPs present in vascular sap might play important roles in banana response to TR4.

Among those, we detected 4 genes of interest. First, the hypersensitive-induced response protein 1 (HIR1) which decreased in abundance in Pahang with no change in Brazilian under TR4 infection HIR1 is known to act as regulators of plant immunity by triggering hypersensitive cell death $[63,64]$. It would suggest that Pahang decreases HIR1 expression to suppress the cell death as a resistance mechanism to TR4 due to Foc as hemibiotroph or necrotroph $[43,65]$.

Ubiquitin involved in the ubiquitination system are key for plant immunity [66]. Ubiquitination is mediated by three step enzymatic cascades, including activating (E1), conjugating (E2) and ligating (E3) enzymes [67]. E3 ubiquitin ligase RING1 gene, CaRING1, played a positive role in pepper (Capsicum annuum) response to microbial pathogens [68]; whereas a homologous triplet of U-box type E3 ubiquitin ligases acted as negative regulators of PTI in Arabidopsis [69]. In the study, E3 with a RING zinc-finger domain 
decreased in abundance only in Pahang response to TR4. However, further studies are needed to prove whether this protein played a negative role in banana response to TR4.

$\mathrm{CHI}$ is an important enzyme of flavonoid pathway involved in the production of phytoalexin [70]. We found a chalcone-flavonone isomerase $(\mathrm{CHI})$ that increased in abundance in Pahang under TR4 infection, as well as in Pahang control compared with Brazilian control.

Finally, GRP containing an RNA recognition motif (RRM) domain was increased in abundance in Pahang response to TR4, but no significant changes in other pairwise comparisons. GRPs act as regulators in diverse cellular processes, including response to stress in plants [71, 72]. Over expressing TaRZ1, a wheat (Triticum aestivum) zinc finger-containing GRP, in Arabidopsis thaliana increased resistance against necrotrophic bacteria Pseudomonas syringae [73]. Although functional validation would be needed to confirm the actions, our results unraveled a list of promising candidate genes to explore.

\section{Pahang specific reaction during TR4 infection}

We previously observed with gene expression on the same genotypes that Pahang exhibits constitutive defense responses before TR4 infection [17]. Proteomic results indicated that the higher number of DEPs (84) is between Pahang and Brazilian without infection. We also identified 26 DEPs by comparing TR4 inoculated Pahang with that of Brazilian, but could find only one protein with increased abundance in both infected bananas. These results show our susceptible and resistant banana genotypes have definitively a different response to TR4 infection. Among the 26 DEPs, 7 proteins have an unknown function but two proteins were highly associated with resistance to pathogen in plant, annotated as carboxylesterase and GDSL esterase/lipase.

Carboxylesterases (CXEs) are implicated in plant defense. A conserved NbCXE inhibited accumulation of Tobacco mosaic virus (TMV) in Nicotiana benthamiana, enhanced plant resistance [74] while constitutive expression of PepEST, a fungus-inducible carboxylesterase in pepper (Capsicum annuum) increased resistance against the hemibiotrophic anthracnose fungus (Colletotrichum gloeosporioides) [75]. In the present study, a CXE protein (Ma06_t34160) was specifically in higher abundance in TR4 inoculated Pahang compared to Brazilian.

Similarly, GDSL esterase/lipases (GLIP) are involved in plant defense against pathogens [76]. Overexpressing GLIP1 in Arabidopsis improved resistance against hemibiotrophic and necrotrophic pathogens $[77,78]$. In the study, a GLIP protein (Ma11_t05100) showed an increased abundance in TR4 inoculated Pahang compared with that of Brazilian. This is consistent with our previous transcriptomic study in which this GLIP gene was also activated by TR4 attack [17].

\section{Conclusions}

To gain an integrated understanding of the changes of banana vascular sap proteins during TR4 infection, we performed a comparative proteomic analysis of vascular sap in resistant diploid 'Pahang' 
and susceptible triploid 'Brazilian' inoculated with TR4 at $14 \mathrm{dpi}$. A total of 1036 proteins were detected in vascular sap, some of these proteins are involved in 'biosynthesis of secondary metabolites', 'environmental adaptation', 'lipid metabolism' and 'signal transduction', which are commonly considered as disease-resistance pathways. Since the vascular sap contained defense-related proteins the constitutive presence of certain proteins could contribute toward resistance. 19 proteins were significantly more abundant after TR4 inoculation, and 26 proteins were only upregulated in the resistant genotype. Of these DEPs, hypersensitive-induced response protein 1 (HIR1) and E3 ubiquitin ligase (E3) decreased in abundance in Pahang with no change in Brazilian under TR4 infection; chalcone isomerase (CHI) and glycine-rich RNA-binding protein (GRP) increased in abundance in Pahang but no significant changes in Brazilian under TR4 infection; carboxylesterase (CXE) and GDSL lipase (GLIP) were specifically in higher abundance in Pahang response to TR4 compared to that of Brazilian. It suggested that these proteins played important roles in bananas against TR4. To our knowledge, this is first report to analyze changes in banana vascular sap proteins response to TR4 to date, which provided insight into resistant mechanisms of banana defense against Fusarium wilt. In the next steps, the function of these proteins will be further validated.

\section{Methods}

\section{Plant inoculation and vascular sap collection}

We selected Musa acuminata 'Pahang' (AA, ITC0609), with official SMTA-2015 from the International Musa Germplasm Transit Centre (ITC), and Musa Cavendish 'Brazilian' (AAA, commercial cultivar in China). Pahang is resistant to TR4, while Brazilian is susceptible to TR4 $[33,79,80]$. The banana inoculation was performed similarly [33] with minor modifications. The roots of banana with 6-8 leaves were cut to $5 \mathrm{~cm}$ and immersed into TR4 conidia suspension for $30 \mathrm{~min}$ at a concentration of $10^{6}$ conidia/mL. The plants were soaked into sterile water as mocks. All plants were transplanted to pots filled with sterile vermiculite, and placed in an artificial climate chamber at $30^{\circ} \mathrm{C}, 80 \%$ humidity, and $8 \mathrm{~h}$ light/16 h dark (Fig. 1a, b). At 14 days post inoculation (dpi), the pseudostems were transected at $0.5 \mathrm{~cm}$ above the corms with a sterile blade (Fig. 1c). After removed the exudate from the cut cells, the vascular sap exuded spontaneously from the remaining pseudostems was collected with a pipette (Fig. 1d-1f). Vascular sap isolated from at least 30 plants was pooled into one independent biological replicate, and three independent biological replicates were conducted. The vascular sap was frozen with liquid nitrogen until protein extraction.

\section{Vascular sap protein extraction}

At least $3 \mathrm{~mL}$ vascular sap was resuspended with benzyltriphenyl phosphonium chloride (BPP) solution (containing $1 \%$ crosslinked polyvinylpyrrolidone, PVPP) in a ratio of $1: 3$, and vortexed at $4{ }^{\circ} \mathrm{C}$ for $10 \mathrm{~min}$. Added equal volume of Tris-saturated phenol, and vortexed at $4^{\circ} \mathrm{C}$ for $10 \mathrm{~min}$. Centrifuged with $12,000 \mathrm{~g}$ at $4^{\circ} \mathrm{C}$ for 20 min to take the phenol phase, and added an equal volume of BPP solution, and vortexed for $10 \mathrm{~min}$ at $4^{\circ} \mathrm{C}$. Centrifuged with $12,000 \mathrm{~g}$ at $4^{\circ} \mathrm{C}$ for $20 \mathrm{~min}$ to collect the phenol phase. The proteins were 
precipitated overnight at $-20^{\circ} \mathrm{C}$ from the phenol phase with pre-cooled ammonium acetate methanol in a ratio of $1: 5$. Centrifuged with $12,000 \mathrm{~g}$ at $4^{\circ} \mathrm{C}$ for 20 min the next day and discarded the supernatant. Washed the pellet twice with $90 \%$ pre-cooled acetone. Used $8 \mathrm{M}$ urea and $1 \%$ sodium dodecyl sulfate (SDS) with a protease inhibitor to dissolve the pellet. Centrifuged with $12,000 \mathrm{~g}$ at $4^{\circ} \mathrm{C}$ for $20 \mathrm{~min}$ to collect the protein supernatant.

\section{Protein Digestion and ITRAQ Labeling}

Protein concentrations were detected using BCA Protein Assay Kit (Pierce, Thermo, USA). Protein digestion was carried out according to the standard procedure. Briefly, took $100 \mu \mathrm{g}$ protein of each sample. Added the final concentration of $10 \mathrm{mM}$ Tris(2-carboxyethyl) phosphine (TCEP) and incubated at $37^{\circ} \mathrm{C}$ for $60 \mathrm{~min}$. Added the final concentration of $40 \mathrm{mM}$ iodoacetamide (IAM) to react at room temperature in the dark for $40 \mathrm{~min}$. Added pre-cooled acetone (acetone: sample volume ratio $=6: 1$ ) to each tube, and incubated at $-20^{\circ} \mathrm{C}$ for $4 \mathrm{~h}$. Centrifuged at $10,000 \mathrm{~g}$ for $20 \mathrm{~min}$, and discarded acetone and took the precipitate. Resuspended the precipitated protein with $100 \mu \mathrm{L} 100 \mathrm{mM}$ tetraethylammonium bromide (TEAB) buffer. Added trypsin solution (1:50) to each tube, and incubated at $37^{\circ} \mathrm{C}$ overnight. The resulting peptide mixture was labeled using the 8-plex iTRAQ reagent (Applied Biosystems, 4390812) according to the manufacturer's instructions [81].

\section{High pH RPLC Separation}

The peptides were separated by high $\mathrm{pH}$ reversed-phase liquid chromatography (RPLC, Acquity Ultra Performance LC, Waters, USA) after resuspended with loading buffer (Ammonium hydroxide solution containing $2 \%$ acetonitrile, $\mathrm{pH} 10$ ). The gradient elution ( $0-2 \mathrm{~min}, 0 \% \mathrm{~B} ; 2-9 \mathrm{~min}, 0 \%-3.8 \% \mathrm{~B} ; 9-27 \mathrm{~min}$, $3.8 \%-24 \%$ B; $27-30 \mathrm{~min}, 24 \%-30 \%$ B; 30-31 min, 30-43\% B; 31-32 min, 43-100\% B; 32-38 min, 100\%-0\% B; 38-58 min, 0\% B) was performed on high pH RPLC column (ACQUITY UPLC BEH C18 Column $1.7 \mu \mathrm{m}, 2.1$ $\mathrm{mm} \times 150 \mathrm{~mm}$, Waters, USA) at a flowrate of $200 \mu \mathrm{L} / \mathrm{min}$ for $58 \mathrm{~min}$ (Phase B $₫ 5 \mathrm{mM}$ Ammonium hydroxide solution containing $98 \%$ acetonitrile, $\mathrm{pH} 10$ ). Twenty fractions were collected from each sample, which was subsequently pooled, resulting in ten total fractions per sample.

\section{Mass spectrometry analysis}

The experiment was conducted on a Q-Exactive (Thermo, USA) mass spectrometer in combination with Easy-nLC 1200. Amount $4 \mu \mathrm{L}$ of each fraction was injected into nano liquid chromatography-tandem mass spectrometry (LC-MS/MS) analysis. Then $2 \mu \mathrm{g}$ peptide mixture was loaded onto the C18-reversed phase column ( $75 \mu \mathrm{m} \times 25 \mathrm{~cm}$, Thermo, USA) in buffer $\mathrm{A}$ ( $2 \%$ acetonitrile with $0.1 \%$ formic acid) and separated with a linear gradient (0-1 min, 0\%-5\% B; 1-63 min, 5\%-23\% B; 63-88 min, 23\%-48\% B; 88-89 min, $48 \%-100 \%$ B; $89-95$ min, $100 \%$ B; $95-100$ min, $100 \%-0 \%$ B; $100-120$ min, 0\% B) of buffer B (80\% acetonitrile with $0.1 \%$ formic acid) at a $300 \mathrm{~nL} / \mathrm{min}$ flow rate. The electrospray voltage of $1.8 \mathrm{kV}$ versus the inlet of the mass spectrometer was used. Q-Exactive mass spectrometer was under operation in the data-dependent mode to switch automatically between MS and MS/MS acquisition. The full-scan MS spectra (m/z 350-1300) survey were monitored with a mass resolution of 70,000, followed by 20 
sequential high-energy collisional dissociation (HCD) MS/MS scans with a resolution of 17,500 . One microscan was recorded with a dynamic exclusion of $18 \mathrm{sec}$, and the MS/MS normalized collision energy was set at 30 .

\section{Functional annotation of peptides}

MS/MS spectra were searched using Proteome Discoverer (Thermo Scientific, Version 2.2) against Musa acuminata database from UniProtKB-SwissProt [82] (http://www.uniprot.org/proteomes/UP000012960) and the decoy database as the following parameters. The highest score for a given peptide mass (best match to that predicted in the database) was used to identify parent proteins. The parameters for protein searching were based on below criteria: tryptic digestion with up to two missed cleavages, carbamidomethylating of cysteines as fixed modification, and oxidation of methionine and protein $\mathrm{N}$ terminal acetylation were considered as variable modifications. False discovery rate (FDR) of peptide identification was set as FDR $\leq 0.01$. In order to support protein identification, a minimum of one unique peptide identification was used.

For 26 genes (Fig. 5e), we added the RefSeq (NCBI) annotation locus codes and the V2 annotation names (column " $C$ " and " $D$ ", respectively) retired at the Banana Genome Hub [83]. Moreover, we added a more informative or alternative functional annotation which was available we added it in the "E" column.

Finally, we checked if one or more paralogs or similar genes were present in Musa acuminata genome V2 [84] (Column "F" and "G", respectively) (Supplementary Table 2).

\section{ITRAQ quantitative proteomics analysis}

The basic information analysis process for iTRAQ quantitative proteomics using the free online platform of Majorbio Cloud Platform (www.majorbio.com). First, the raw mass spectra generated by the mass spectrometer were subjected to the peak identification. Secondly, the UniProtKB-SwissProt reference proteomic database of banana (http://www.uniprot.org/proteomes/UP000012960) was established to identify peptides and proteins. All identified proteins were functional annotated using Cluster of Orthologous Groups of proteins (COG, http://eggnogdb.embl.de/\#/app/home), Gene Ontology (GO, http://www.geneontology.org/) and Kyoto Encyclopedia of Genes and Genomes (KEGG, http://www.genome.jp/kegg/) with E-value $\leq 1 \times 10^{-5}$ and identity $\geq 0.98$. The DEPs were identified with fold change $>1.2$ (upregulation), fold change $<0.83$ (downregulation) and $P$ value $<0.05$ [23], and analyzed including DEPs Venn and expression pattern analysis.

\section{Abbreviations}

Foc TR4: Fusarium oxysporum f. sp. cubense tropical race 4; DEPs: Differential expression proteins; HIR1: Hypersensitive-induced response protein 1; E3: E3 ubiquitin ligase; $\mathrm{CHI}$, Chalcone isomerase; GRP: glycinerich RNA-binding protein; CXE: Carboxylesterase; GLIP: GDSL lipase; RGA2: Resistance gene analogue 2; dpi: Days post inoculation; BPP: Benzyltriphenylphosphonium chloride; PVPP: Crosslinked polyvinylpyrrolidone; SDS: Sodium dodecyl sulfate; BCA: Bicinchoninic; iTRAQ: Isobaric tags for relative 
and absolute quantitation; TCEP: Tris(2-carboxyethyl)phosphine; IAM: lodoacetamide; TEAB:

Tetraethylammonium bromide; RPLC: Reversed-phase liquid chromatography; LC-MS/MS: Liquid chromatography-tandem mass spectrometry; HCD: High energy collisional dissociation; FDR: False discovery rate; COG: Cluster of Orthologous Groups; GO: Gene Ontology; KEGG: Kyoto Encyclopedia of Genes and Genomes; MAPK: Mitogen-activated protein kinase; NDPK: Nucleoside diphosphate kinase; HR: Hypersensitive response; SA: Salicylic acid; JA: Jasmonic acid; PR1: Pathogenesis-related protein 1; SAR: Systemic acquired resistance; COI1: Coronatine-insensitive protein homolog; ISR: Induced systemic resistance; PAMP: Pathogen-associated molecular pattern; PRRs: Pathogen recognition receptors; PTI: PAMP-triggered immunity; ROS: Reactive oxygen species; CDPK: Calcium-dependent protein kinase; CaMCML: Calcium-binding protein CML; PAL: Phenylalanine ammonia-lyase; C4L: 4-coumarate-CoA ligase; CCR: Cinnamoyl-CoA reductase; CAD: Cinnamyl alcohol dehydrogenase; POD: Peroxidase; CCOAOMT: Caffeoyl-CoA 0-methyltransferase; CHS: Chalcone synthase; F3'5'H: Flavonoid 3',5'hydroxylase; DFR: Dihydroflavonol-4-reductase; LACS: Long chain acyl-CoA synthetases; PLDa1: Phospholipase D a1; RRM: RNA recognition motif; TMV: Tobacco mosaic virus.

\section{Declarations}

\section{Ethics approval and consent to participate}

Not applicable.

\section{Consent for publication}

The authors confirm that the work described has not been published before. All authors read and approved the final manuscript.

\section{Availability of data and materials}

Musa acuminata 'Pahang' (AA, ITC0609) is imported with official SMTA-2015 from the International Musa Germplasm Transit Centre (ITC). The mass spectrometry proteomics data have been deposited to the ProteomeXchange Consortium (http://proteomecentral.proteomexchange.org) via the iProX partner repository [85] with the dataset identifier PXD018261.

\section{Competing interests}

The authors declare that no competing interests exist.

\section{Funding}

The Yunling Scholar Programme of Yunnan Provincial Government (YNWR-YLXZ-2018-018), the National Natural Science Foundation of China (NSFC31560505), the Science and Technology Department of Yunnan Provincial Government (2018BB016), Program for the Innovative Research Team of Yunnan Province (2020-2022), Yunnan University of Chinese Medicine (30270104879), the CGIAR Research 
Program (CRP) on Roots, Tubers and Bananas (RTB) provide project research funds and analysis, and interpretation of data and in writing the manuscript.

\section{Authors' contributions}

LZ and SJZ: conceptualization. LZ: performed the experiments, analyzed the data and wrote the paper. $\mathrm{LL}, \mathrm{SL}, \mathrm{TB}, \mathrm{SX}, \mathrm{HF}, \mathrm{KY}$, and PH: analyzed the data. MR, AC and SC: provided feedback on data analyses and reviewed the manuscript. YW and WT proof writing. SJZ: conceived and funding acquisition, designed the experiments and proof writing. All authors have read and approved the manuscript.

\section{Acknowledgements}

We thank Shanghai Majorbio Bio-pharm Technology Co., Ltd. for assistance with the RNA-Seq experiments and data analysis.

\section{References}

1. Ploetz RC, Evans EA. The future of global banana production. Horticultural reviews. 2015;43:311-352.

2. Ploetz RC. Fusarium wilt of banana. Phytopathology. 2015;105:1512-1521.

3. Ghag SB, Shekhawat UKS, Ganapathi TR. Fusarium wilt of banana: biology, epidemiology and management. International Journal of Pest Management. 2015;61:250-263.

4. Butler D. Fungus threatens top banana. Nature. 2013;504:195-196.

5. Dita M, Barquero M, Heck D, Mizubuti ES, Staver CP. Fusarium wilt of banana: current knowledge on epidemiology and research needs toward sustainable disease management. Frontiers in plant science. 2018;9:1468.

6. Zheng S-J, García-Bastidas FA, Li X, Zeng L, Bai T, Xu S, Yin K, Li H, Fu G, Yu Y et al. New geographical insights of the latest expansion of Fusarium oxysporum f. sp. cubense tropical race 4 into the Greater Mekong Subregion. Frontiers in Plant Science. 2018;9:457.

7. Ozarslandan M, AKGÜL DS. First report of Fusarium oxysporum f. sp. cubense race 4 causing Fusarium wilt disease of banana in Turkey. Plant Disease. 2020;104:974.

8. Maymon M, Sela N, Shpatz U, Galpaz N, Freeman S. The origin and current situation of Fusarium oxysporum f. sp. cubense tropical race 4 in Israel and the Middle East. Scientific Reports. 2020;10:111.

9. Ploetz RC. Fusarium wilt of banana is caused by several pathogens referred to as Fusarium oxysporumf. sp. cubense. Phytopathology. 2006;96:653-656.

10. Stokstad E. Banana fungus puts Latin America on alert. Science. 2019;365:207-208.

11. Garcia-Bastidas F, Quintero-Vargas C, Ayala-Vasquez M, Seidl M, Schermer T, Santos-Paiva M, Noguera AM, Aguilera-Galvez C, Wittenberg A, Sørensen A. First report of Fusarium wilt Tropical Race 4 in Cavendish bananas caused by Fusarium odoratissimum in Colombia. Plant Disease. 2020;104:994. 
12. Ploetz RC. Management of Fusarium wilt of banana: A review with special reference to tropical race 4. Crop Protection. 2015;73:7-15.

13. Siamak SB, Zheng S. Banana Fusarium wilt (Fusarium oxysporum f. sp. cubense) control and resistance, in the context of developing wilt-resistant bananas within sustainable production systems. Horticultural Plant Journal. 2018;4:208-218.

14. Ghag SB, Ganapathi TR. Genetically modified bananas: To mitigate food security concerns. Scientia Horticulturae. 2017;214:91-98.

15. Maxmen A. CRISPR might be the banana's only hope against a deadly fungus. Nature. 2019;574:15.

16. Pua TL, Tan TT, Jalaluddin NS, Othman RY, Harikrishna JA. Genetically engineered bananas-From laboratory to deployment. Annals of Applied Biology. 2019;175:282-301.

17. Zhang L, Cenci A, Rouard M, Zhang D, Wang Y, Tang W, Zheng SJ. Transcriptomic analysis of resistant and susceptible banana corms in response to infection by Fusarium oxysporum $\mathrm{f}$. sp. cubense tropical race 4. Sci Rep. 2019;9:8199.

18. Jansen RC, Nap JP, Mlynarova L. Errors in genomics and proteomics. Nature biotechnology. 2002;20:19.

19. Campos NA, Swennen R, Carpentier SC. The plantain proteome, a focus on allele specific proteins obtained from plantain fruits. Proteomics. 2018;18:1700227.

20. Carpentier SC, Coemans B, Podevin N, Laukens K, Witters E, Matsumura H, Terauchi R, Swennen R, Panis B. Functional genomics in a non-model crop: transcriptomics or proteomics? Physiologia plantarum. 2008;133:117-130.

21. Lu Y, Liao D, Pu J, Qi Y, Xie Y. Proteome analysis of resistant and susceptible Cavendish banana roots following inoculation with Fusarium oxysporum f. sp. cubense. Physiological and molecular plant pathology. 2013;84:163-171.

22. Li X, Bai T, Li Y, Ruan X, Li H. Proteomic analysis of Fusarium oxysporum f. sp. cubense tropical race 4-inoculated response to Fusarium wilts in the banana root cells. Proteome Science 2013;11.

23. Dong H, Li Y, Fan H, Zhou D, Li H. Quantitative proteomics analysis reveals resistance differences of banana cultivar 'Brazilian' to Fusarium oxysporum f. sp. cubense races 1 and 4. Journal of proteomics. 2019;203:103376.

24. Fernandez-Garcia N, Hernandez M, Casado-Vela J, Bru R, Elortza F, Hedden P, Olmos E. Changes to the proteome and targeted metabolites of xylem sap in Brassica oleracea in response to salt stress. Plant Cell and Environment. 2011;34:821-836.

25. Satoh S. Organic substances in xylem sap delivered to above-ground organs by the roots. Journal of Plant Research. 2006;119:179-187.

26. Carella P, Wilson DC, Kempthorne CJ, Cameron RK. Vascular sap proteomics: Providing insight into long-distance signaling during stress. Frontiers in Plant Science. 2016;7:651.

27. Rep M, Dekker HL, Vossen JH, de Boer AD, Houterman PM, Speijer D, Back JW, de Koster CG, Cornelissen BJC. Mass spectrometric identification of isoforms of PR proteins in xylem sap of 
fungus-infected tomato. Plant Physiology. 2002;130:904-917.

28. Houterman PM, Speijer D, Dekker HL, de Koster CG, Cornelissen BJ, Rep M. The mixed xylem sap proteome of Fusarium oxysporum-infected tomato plants. Molecular plant pathology. 2007;8:215221.

29. Gawehns F, Ma L, Bruning O, Houterman PM, Boeren S, Cornelissen BJC, Rep M, Takken FLW. The effector repertoire of Fusarium oxysporum determines the tomato xylem proteome composition following infection. Frontiers in Plant Science. 2015;6.

30. de Lamo FJ, Constantin ME, Fresno DH, Boeren S, Rep M, Takken FL. Xylem sap proteomics reveals distinct differences between $\mathrm{R}$ gene-and endophyte-mediated resistance against Fusarium wilt disease in tomato. Frontiers in microbiology. 2018;9:2977.

31. Pu Z, Ino Y, Kimura Y, Tago A, Shimizu M, Natsume S, Sano Y, Fujimoto R, Kaneko K, Shea DJ et al. Changes in the proteome of xylem sap in Brassica oleracea in response to Fusarium oxysporum stress. Frontiers in Plant Science. 2016;7.

32. Poon NK, Teo CH, Othman RY. Differential gene expression analysis of Secreted in Xylem (SIX) genes from Fusarium oxysporum f. sp. cubense tropical race 4 in Musa acuminata cv. Berangan and potential application for early detection of infection. Journal of General Plant Pathology. 2020;86:1323.

33. Zhang L, Yuan T, Wang Y, Zhang D, Bai T, Xu S, Wang Y, Tang W, Zheng S-J. Identification and evaluation of resistance to Fusarium oxysporum f. sp. cubense tropical race 4 in Musa acuminata Pahang. Euphytica. 2018;214.

34. Benhamou N. Elicitor-induced plant defence pathways. Trends in Plant Science. 1996;1(7):233-240.

35. Baker B, Zambryski P, Staskawicz B, Dinesh-Kumar S. Signaling in plant-microbe interactions. Science. 1997;276:726-733.

36. Pandey D, Rajendran SRCK, Gaur M, Sajeesh P, Kumar A. Plant defense signaling and responses against necrotrophic fungal pathogens. Journal of Plant Growth Regulation. 2016;35:1159-1174.

37. Meng X, Zhang S. MAPK cascades in plant disease resistance signaling. Annual review of phytopathology. 2013;51:245-266.

38. Pitzschke A, Schikora A, Hirt H. MAPK cascade signalling networks in plant defence. Current opinion in plant biology. 2009;12:421-426.

39. Reymond P, Farmer EE. Jasmonate and salicylate as global signals for defense gene expression. Current opinion in plant biology. 1998;1:404-411.

40. Kunkel BN, Brooks DM. Cross talk between signaling pathways in pathogen defense. Curr Opin Plant Biol. 2002;5:325-331.

41. Pieterse CM, Van der Does D, Zamioudis C, Leon-Reyes A, Van Wees SC. Hormonal modulation of plant immunity. Annual review of cell and developmental biology. 2012;28:489-521.

42. Glazebrook J. Contrasting mechanisms of defense against biotrophic and necrotrophic pathogens. In: Annual Review of Phytopathology. vol. 43; 2005;205-227. 
43. Lo Presti L, Lanver D, Schweizer G, Tanaka S, Liang L, Tollot M, Zuccaro A, Reissmann S, Kahmann R. Fungal effectors and plant susceptibility. In: Annual Review of Plant Biology, Vol 66. Edited by Merchant SS, vol. 66; 2015;513-545.

44. Sun D, Lu X, Hu Y, Li W, Hong K, Mo Y, Cahill DM, Xie J. Methyl jasmonate induced defense responses increase resistance to Fusarium oxysporum f. sp. cubense race 4 in banana. Scientia Horticulturae. 2013;164:484-491.

45. Wang Z, Jia C, Li J, Huang S, Xu B, Jin Z. Activation of salicylic acid metabolism and signal transduction can enhance resistance to Fusarium wilt in banana (Musa acuminata L. AAA group, cv. Cavendish). Functional \& Integrative Genomics. 2015;15:47-62.

46. Van Loon LC, Van Strien EA. The families of pathogenesis-related proteins, their activities, and comparative analysis of PR-1 type proteins. Physiological and Molecular Plant Pathology. 1999;55:85-97.

47. Feys BJ, Parker JE. Interplay of signaling pathways in plant disease resistance. Trends in Genetics. 2000;16:449-455.

48. Jones JDG, Dangl JL. The plant immune system. Nature. 2006;444:323-329.

49. Monaghan J, Zipfel C. Plant pattern recognition receptor complexes at the plasma membrane. Current opinion in plant biology. 2012;15:349-357.

50. Zhang L, Du L, Poovaiah B. Calcium signaling and biotic defense responses in plants. Plant signaling \& behavior. 2014;9:e973818.

51. Schulz P, Herde M, Romeis T. Calcium-dependent protein kinases: hubs in plant stress signaling and development. Plant physiology. 2013;163:523-530.

52. Piasecka A, Jedrzejczak-Rey N, Bednarek P. Secondary metabolites in plant innate immunity: conserved function of divergent chemicals. New Phytologist. 2015;206:948-964.

53. Sun J, Zhang J, Fang H, Peng L, Wei S, Li C, Zheng S, Lu J. Comparative transcriptome analysis reveals resistance-related genes and pathways in Musa acuminata banana 'Guijiao 9' in response to Fusarium wilt. Plant Physiology and Biochemistry. 2019;141:83-94.

54. Yang Q, He Y, Kabahuma M, Chaya T, Kelly A, Borrego E, Bian Y, El Kasmi F, Yang L, Teixeira P et al. A gene encoding maize caffeoyl-CoA 0-methyltransferase confers quantitative resistance to multiple pathogens. Nature Genetics. 2017;49:1364-1372.

55. Dao T, Linthorst H, Verpoorte R. Chalcone synthase and its functions in plant resistance. Phytochemistry Reviews. 2011;10:397.

56. Schijlen EG, De Vos CR, van Tunen AJ, Bovy AG. Modification of flavonoid biosynthesis in crop plants. Phytochemistry. 2004;65:2631-2648.

57. Shah J. Lipids, lipases, and lipid-modifying enzymes in plant disease resistance. Annu Rev Phytopathol. 2005;43:229-260.

58. Lim G-H, Singhal R, Kachroo A, Kachroo P. Fatty acid-and lipid-mediated signaling in plant defense. Annual review of Phytopathology. 2017;55:505-536. 
59. Schnurr J, Shockey J. The acyl-CoA synthetase encoded by LACS2 is essential for normal cuticle development in Arabidopsis. The Plant Cell. 2004;16:629-642.

60. Tang D, Simonich MT, Innes RW. Mutations in LACS2, a long-chain acyl-coenzyme A synthetase, enhance susceptibility to avirulent Pseudomonas syringae but confer resistance to Botrytis cinerea in Arabidopsis. Plant physiology. 2007;144:1093-1103.

61. Li J, Wang X. Phospholipase D and phosphatidic acid in plant immunity. Plant science. 2019;279:4550.

62. Serra-Soriano M, Antonio Navarro J, Genoves A, Pallas V. Comparative proteomic analysis of melon phloem exudates in response to viral infection. Journal of Proteomics. 2015;124:11-24.

63. Choi HW, Kim YJ, Hwang BK. The hypersensitive induced reaction and leucine-rich repeat proteins regulate plant cell death associated with disease and plant immunity. Molecular plant-microbe interactions. 2011;24:68-78.

64. Zhou L, Cheung M-Y, Li M-W, Fu Y, Sun Z, Sun S-M, Lam H-M. Rice hypersensitive induced reaction protein 1 (OsHIR1) associates with plasma membrane and triggers hypersensitive cell death. BMC plant biology. 2010;10:290.

65. Laluk K, Mengiste T. Necrotroph attacks on plants: wanton destruction or covert extortion? The Arabidopsis Book. 2010;8:e0136.

66. Trujillo M, Shirasu K. Ubiquitination in plant immunity. Current opinion in plant biology. 2010; 13:402408.

67. Vierstra RD. The ubiquitin-26S proteasome system at the nexus of plant biology. Nature Reviews Molecular Cell Biology. 2009;10:385.

68. Lee DH, Choi HW, Hwang BK. The pepper E3 ubiquitin ligase RING1 gene, CaRING1, is required for cell death and the salicylic acid-dependent defense response. Plant Physiology. 2011; 156:2011-2025.

69. Trujillo M, Ichimura K, Casais $C$, Shirasu K. Negative regulation of PAMP-triggered immunity by an E3 ubiquitin ligase triplet in Arabidopsis. Current Biology. 2008;18:1396-1401.

70. Yin Y-c, Zhang X-d, Gao Z-q, Hu T, Liu Y. The research progress of chalcone isomerase $(C H /)$ in plants. Molecular biotechnology. 2019;61:32-52.

71. Jung HJ, Park SJ, Kang H. Regulation of RNA metabolism in plant development and stress responses. Journal of Plant Biology. 2013;56:123-129.

72. Kang H, Park SJ, Kwak KJ. Plant RNA chaperones in stress response. Trends in plant science. 2013; 18:100-106.

73. Xu T, Lee HJ, Sy ND, Kang H. Wheat (Triticum aestivum) zinc finger-containing glycine-rich RNAbinding protein TaRZ1 affects plant growth and defense response in Arabidopsis thaliana. Plant Growth Regulation. 2015;76:243-250.

74. Guo S, Wong S-M. A conserved carboxylesterase inhibits Tobacco mosaic virus (TMV) accumulation in Nicotiana benthamiana plants. Viruses. 2020;12:195. 
75. Ko M, Cho JH, Seo H-H, Lee H-H, Kang H-Y, Nguyen TS, Soh HC, Kim YS, Kim J-I. Constitutive expression of a fungus-inducible carboxylesterase improves disease resistance in transgenic pepper plants. Planta. 2016;244:379-392.

76. Lee H-J, Park OK. Lipases associated with plant defense against pathogens. Plant Science. 2019; 279:51-58.

77. Oh IS, Park AR, Bae MS, Kwon SJ, Kim YS, Lee JE, Kang NY, Lee S, Cheong H, Park OK. Secretome analysis reveals an Arabidopsis lipase involved in defense against Alternaria brassicicola. The Plant Cell. 2005;17:2832-2847.

78. Kwon SJ, Jin HC, Lee S, Nam MH, Chung JH, Kwon SI, Ryu CM, Park OK. GDSL lipase-like 1 regulates systemic resistance associated with ethylene signaling in Arabidopsis. The Plant Journal. 2009;58:235-245.

79. D'Hont A, Denoeud F, Aury J-M, Baurens F-C, Carreel F, Garsmeur O, Noel B, Bocs S, Droc G, Rouard M et al. The banana (Musa acuminata) genome and the evolution of monocotyledonous plants. Nature. 2012;488:213-217.

80. Zuo C, Deng G, Li B, Huo H, Li C, Hu C, Kuang R, Yang Q, Dong T, Sheng $\mathrm{O}$ et al. Germplasm screening of Musa spp. for resistance to Fusarium oxysporum f. sp. cubense tropical race 4 (Foc TR4). Eur J Plant Pathol. 2018;151:723-734.

81. Aggarwal K, Choe LH, Lee KH. Shotgun proteomics using the iTRAQ isobaric tags. Briefings in functional genomics \& proteomics. 2006;5:112-120.

82. Magrane M. UniProt Knowledgebase: a hub of integrated protein data. Database 2011, 2011(2011).

83. Droc G, Larivière D, Guignon V, Yahiaoui N, This D, Garsmeur O, Dereeper A, Hamelin C, Argout X, Dufayard JF et al. The banana genome hub. Database : the journal of biological databases and curation. 2013;2013:bat035.

84. Martin G, Baurens F-C, Droc G, Rouard M, Cenci A, Kilian A, Hastie A, Dolezel J, Aury J-M, Alberti A et al. Improvement of the banana "Musa acuminata" reference sequence using NGS data and semiautomated bioinformatics methods. Bmc Genomics. 2016;17.

85. Ma J, Chen T, Wu S, Yang C, Bai M, Shu K, Li K, Zhang G, Jin Z, He F. iProX: an integrated proteome resource. Nucleic Acids Research. 2019;47:1211-1217.

\section{Figures}



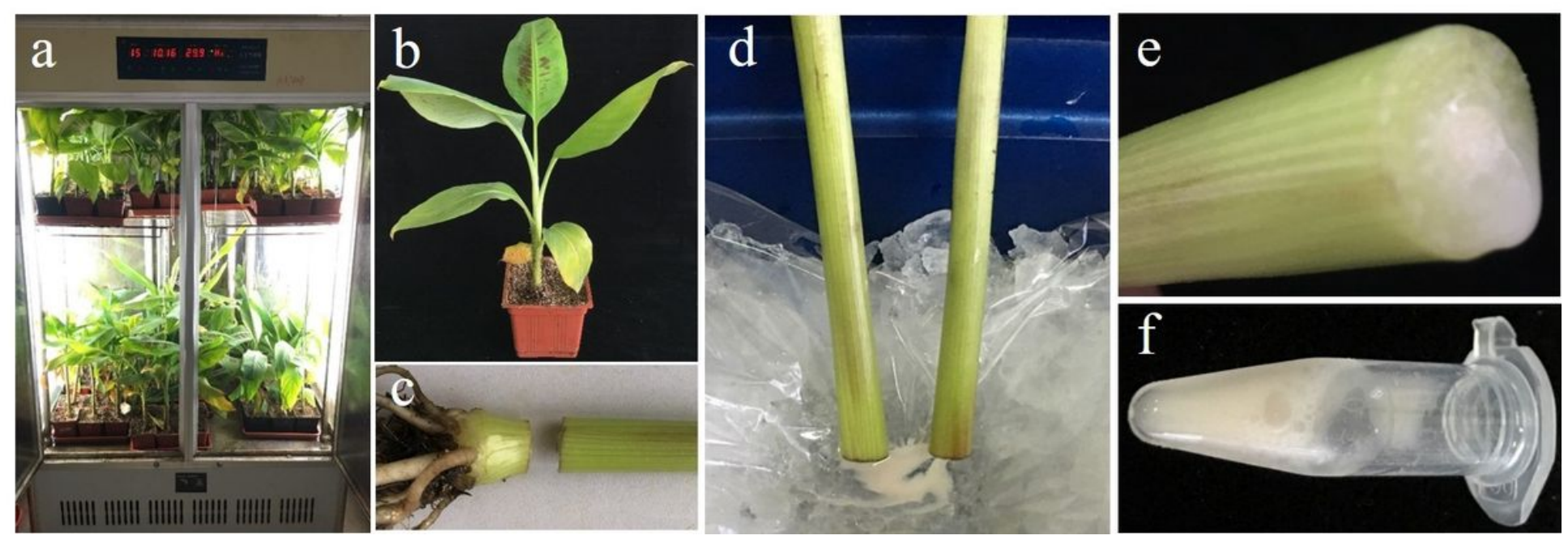

Figure 1

Banana vascular sap collection. (a) The inoculated banana plants were placed in an artificial climate chamber. (b) Banana with 6-8 leaves were inoculated with TR4 and were transplanted to plastic pots filled with sterile vermiculite. (c) The pseudostem of banana was transected at $0.5 \mathrm{~cm}$ above the corms. (d) The transected pseudostem of banana was placed on the ice to collect vascular sap. (e) Banana vascular sap exuded spontaneously from the transected pseudostem. (f) Banana vascular sap was collected in a pipette. 


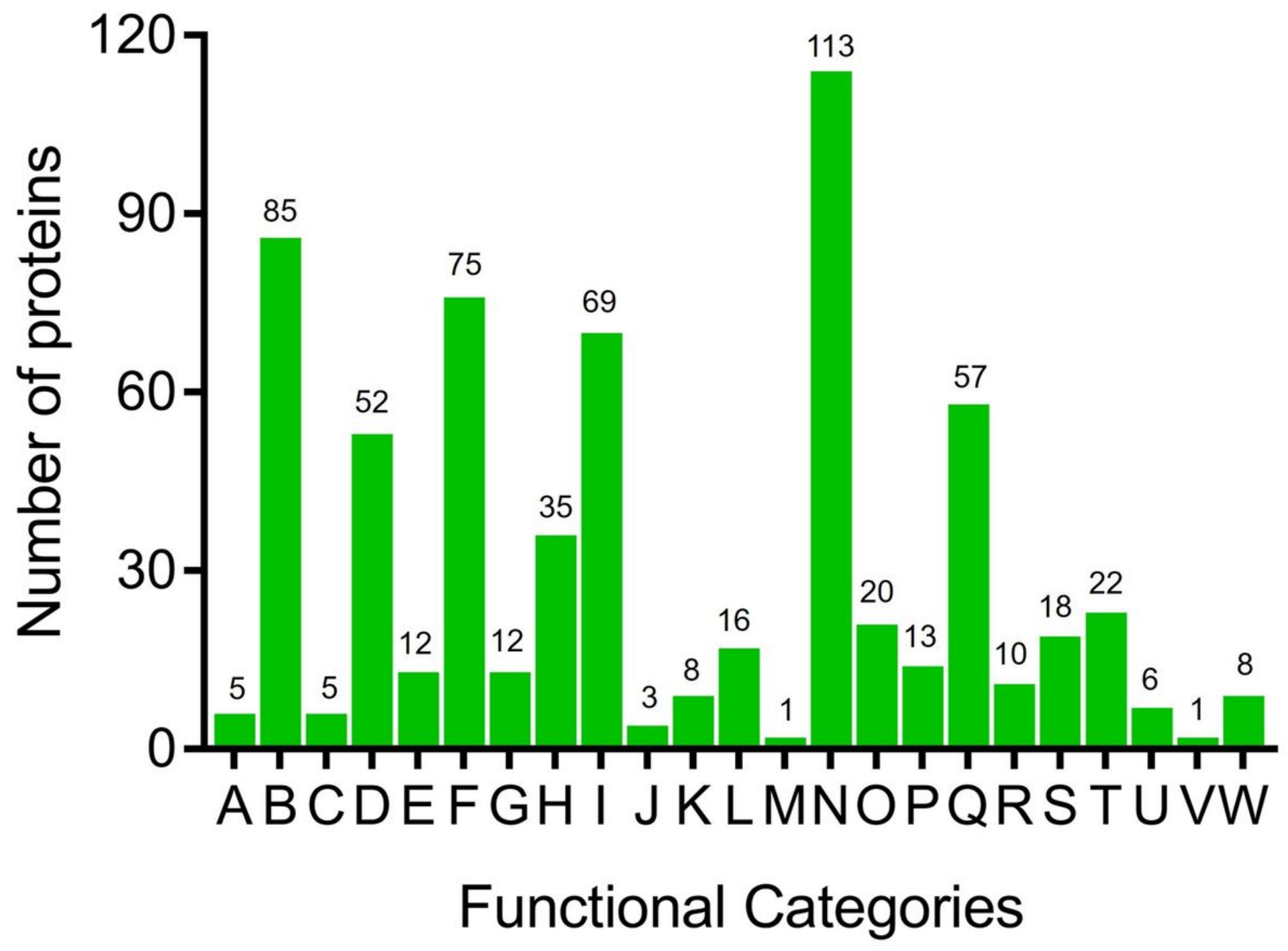

Figure 2

COG functional classification of all identified proteins. 


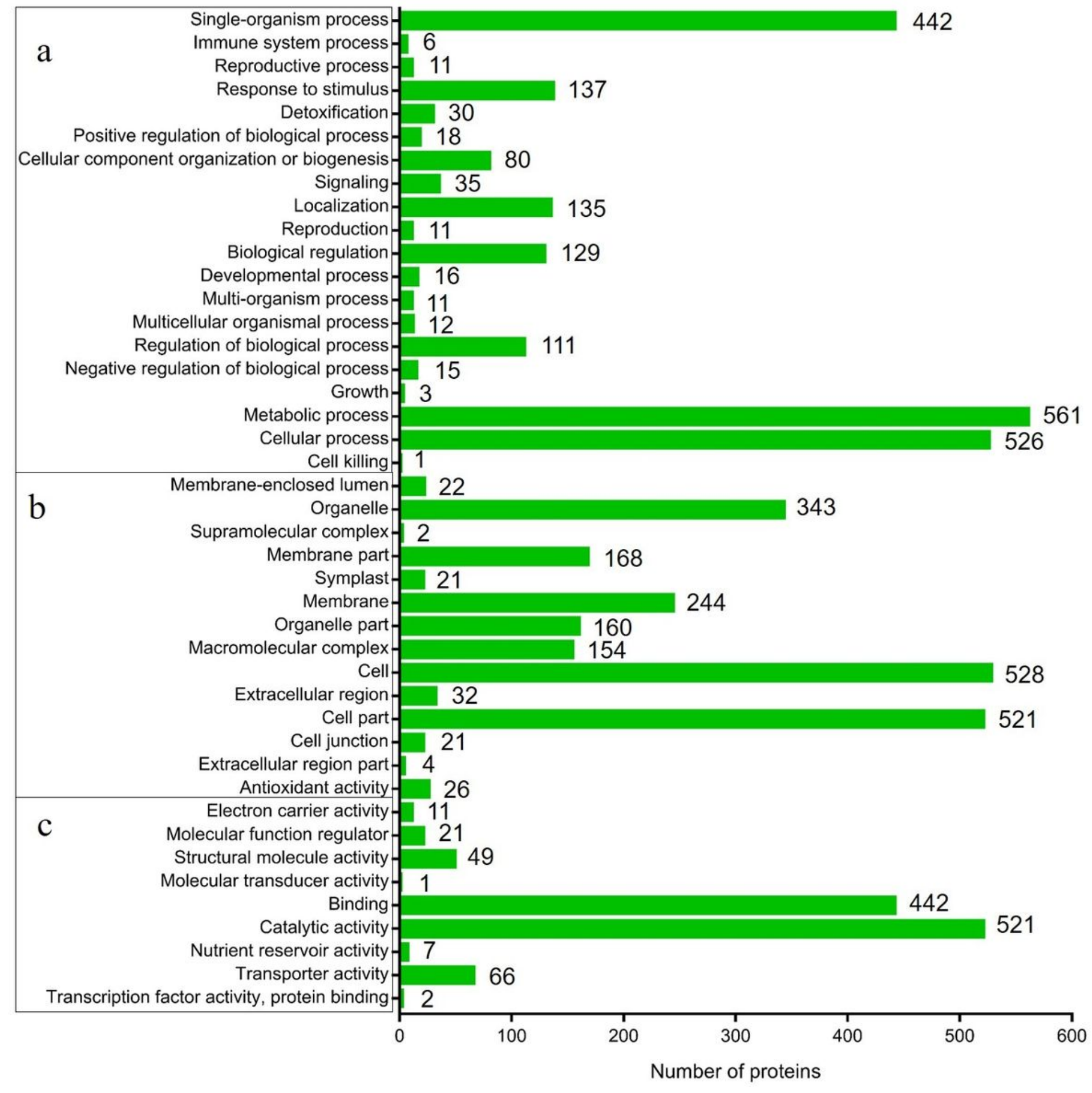

Figure 3

GO functional classification of all identified proteins. (a) Biological process. (b) Cellular component. (c) Molecular function. 


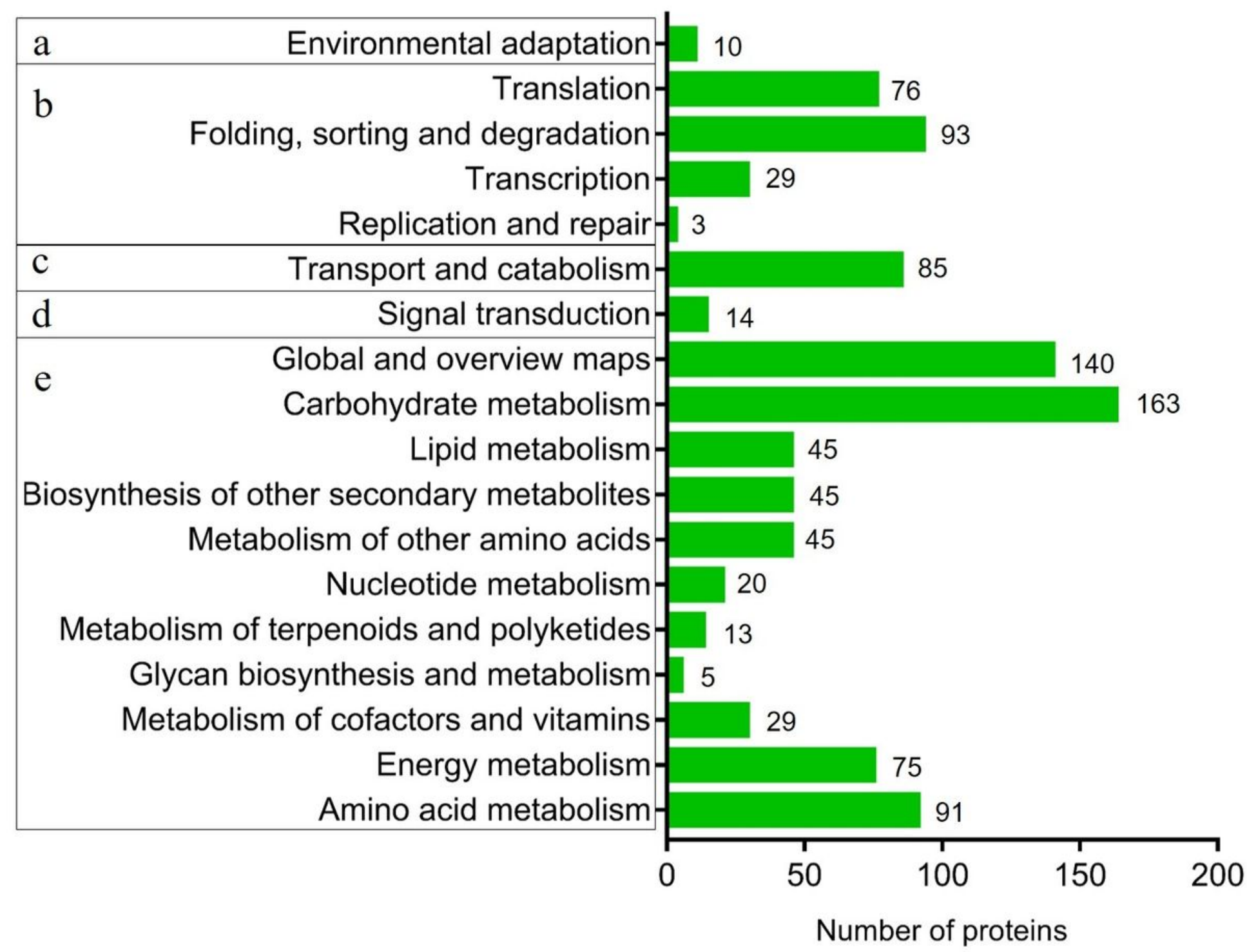

Figure 4

KEGG functional classification of all identified proteins. (a) Organismal systems. (b) Genetic information processing. (c) Cellular processes. (d) Environmental information processing. (e) Metabolism. 


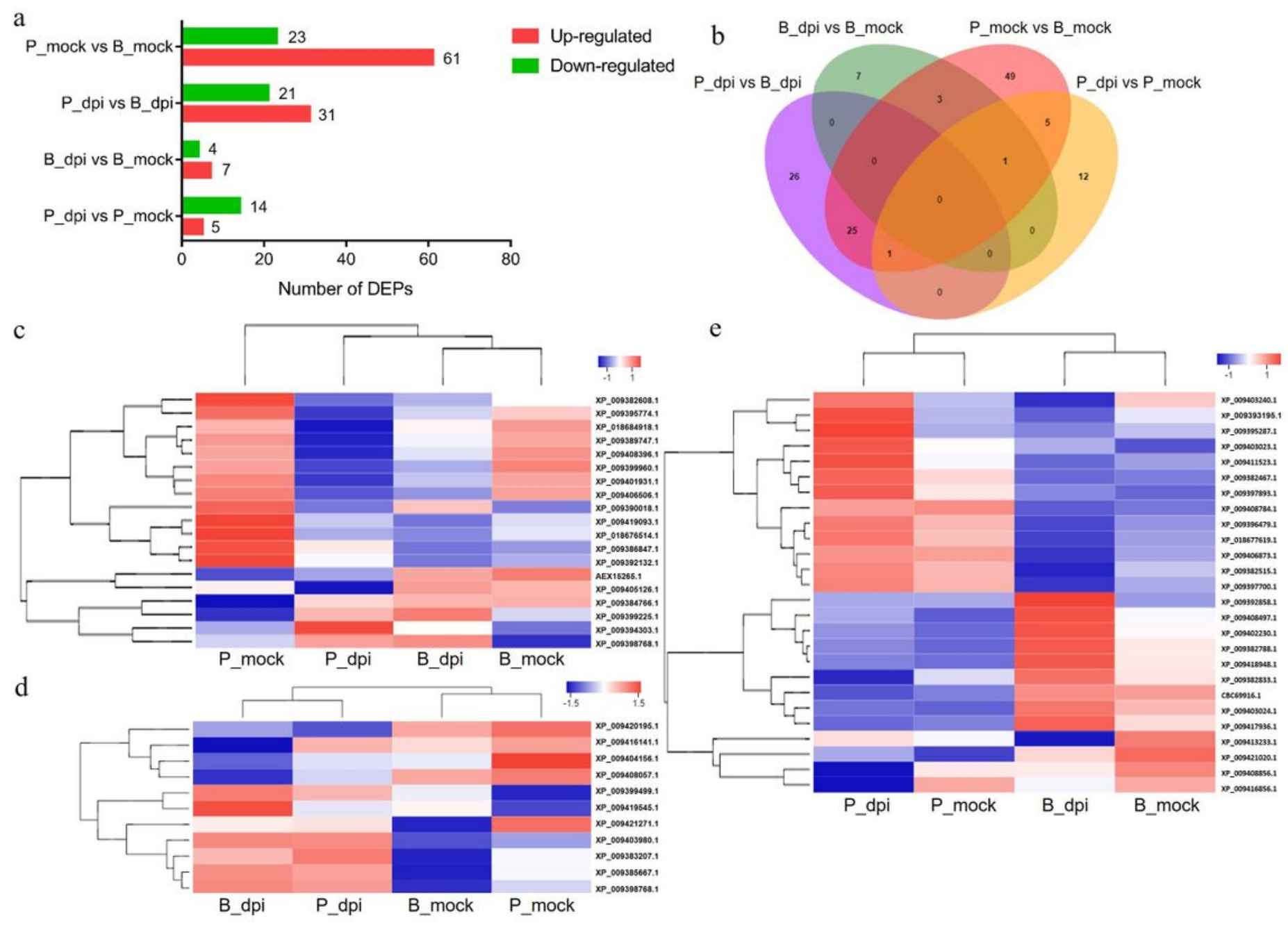

Figure 5

Number of DEPs, Venn diagram and hierarchical cluster analysis of DEPs. (a) Number of identified proteins in the 4 pairwise comparisons between the 2 resistant and susceptible genotypes. (b) Venn diagram analysis of identified in 4 pairwise comparisons. (c) DEPs identified by comparing TR4 inoculated Pahang with mock samples (P_dpi vs P_mock). (d) DEPs identified by comparing TR4 inoculated Brazilian with mock samples (B_dpi vs B_mock). (e) DEPs identified by comparing TR4 inoculated Pahang with that of Brazilian, and the DEPs, in Pahang mock samples versus that of Brazilian, were excluded ('P_dpi vs B_dpi'-'P_mock vs B_mock'). The protein abundances were normalized to the same total peptide amount per channel and scaled.

\section{Supplementary Files}

This is a list of supplementary files associated with this preprint. Click to download.

- SupplementaryTable1.xls 
- SupplementaryTable2.xls

Page 24/24 